\title{
INVESTIGATIONS OF THE SURFACE CONDUCTIVITY OF SILICON DIOXIDE AND METHODS TO REDUCE IT
}

\author{
J.A. VOORTHUYZEN, K. KESKIN and P. BERGVELD \\ Twente University of Technology, P.O. Box 217, 7500 AE Enschede, The Netherlands
}

Received 21 October 1986; accepted for publication 8 April 1987

In this paper we describe our investigations of the electrical conductivity of the silicon dioxide-air interface. It appears that this conductivity is caused by the adsorption of water vapour on the oxide surface and strongly depends on the relative humidity of the surrounding air. Considering this fact we have decided to investigate the possibility to reduce the surface conductivity by means of a chemical modification of the oxide surface, which reduces the adsorption of water vapour on it. To measure the conductivity we have used a so-called open-gate FET structure. The performance characteristics of this structure and the experimental results obtained with it are presented. We have found that by using silane agents like HMDS and DCDMS the surface conductivity of silicon dioxide can be reduced with at least a factor 1000 and 10000 respectively.

\section{Introduction}

Investigating the application of the metal electret air oxide silicon structure as a pressure sensor we found that its performance characteristics were limited by a considerable drift [1], which manifests itself as a low-frequency change of the sensor output, although the measured pressure remains constant. Studying the literature, dealing with other sensors with an electrically floating oxide-air interface, we noticed that the performance characteristics of these devices are also limited by a more or less significant drift. Examples are the resonant gate transistor [2], the direct gate field-effect transistor [3], the biomedical piezoresistive pressure sensor [4], and the movable-gate-field-effect structure [5]. Therefore we have decided to investigate the electrical properties (especially the conductivity) of the oxide-air interface in more detail.

Shockley et al. have already shown that mobile charges present on an oxide-air interface move, due to an applied lateral electric field. They found that the surface conductivity strongly depends on the partial pressure of polar gases in the surrounding air, such as ammonia and water [6]. As water-vapour is the most important polar gas present in environmental air, we shall pay special attention to its influence on the surface conductivity. 
In section 2 the theoretical background of water-vapour adsorption on a silicon dioxide surface, the dependence of the surface conductivity on the relative humidity of the surrounding air and the relation between surface potential variations and surface conductivity will be discussed.

In section 3 the test structure is presented which has been used to measure the surface conductivity. In the same section it will be shown how the experimental results can be used to quantify the surface conductivity. Using the theoretical description given in section 2 , the appropriate passivation methods for the oxide surface are discussed in section 4. In section 5 the conclusions are presented.

\section{Theoretical considerations}

\subsection{Adsorption of water vapour on $\mathrm{SiO}_{2}$}

Two types of water-vapour adsorption occur in sequence at the $\mathrm{SiO}_{2}$-air interface. The chemisorption of water vapour first modifies the $\mathrm{SiO}_{2}$ surface, resulting in a surface with silanol groups $(\mathrm{Si}-\mathrm{OH})$. The second type of adsorption, physisorption, occurs on these silanol groups. A schematic illustration of the water-vapour adsorption is given in fig. 1 .

At room temperature the physisorption is a reversible function of the relative humidity of the surrounding air, while the chemisorption appears to be irreversible. In the temperature range of $180-400^{\circ} \mathrm{C}$ the chemisorption is also reversible, but at temperatures above $400^{\circ} \mathrm{C}$ the surface structure changes and the originally chemisorbed molecules desorb, after which chemisorption can no longer take place at these temperatures. In the succeeding discussion we shall only consider the physisorption [7].

Awakuni and Calderwood investigated the adsorption of water vapour on the $\mathrm{SiO}_{2}$ surface. They measured the amount of adsorbed water as a function of the partial vapour pressure at a constant temperature. It appeared that this so-called adsorption isotherm can be described very well by the BET adsorp-

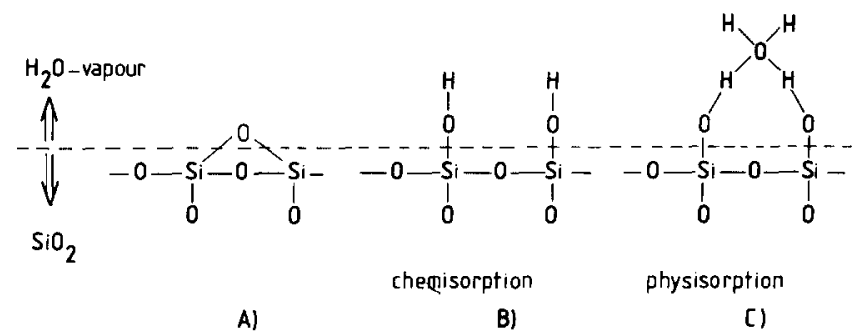

Fig. 1. Schematic presentation of water-vapour adsorption mechanisms on an $\mathrm{SiO}_{2}$ surface. 
tion theory [8]. The BET theory, postulated by Brunauer, Emmet and Teller, assumes that a statistical distribution occurs of surface parts without any adsorbed molecules, parts with one monolayer, parts with two monolayers and so on. It can be shown that for an untreated silicon dioxide surface the coverage ratio $R_{\mathrm{c}}$, defined as the relative part of the surface covered with one or more monolayers of water, can be written as a function of the relative humidity [9]:

$R_{\mathrm{c}}=\frac{C h}{1+(C-1) h}$,

with $h$ the relative humidity and $C$ a dimensionless constant. Using the numerical results of adsorption measurements performed by Young [10] we have estimated the value of $C$. It appeared that it must be in the range of $0.01-0.06$. Castagne et al. [9] determined $C$ from the surface conductivity measurements as a function of relative humidity. They found $C$ to be approximately 0.04 . In the following sections we shall assume that $C=0.05$. It should be noted that eq. (1) is valid only for an untreated surface.

\subsection{A discourse on the surface conductivity}

According to the literature, dealing with the surface conductivity of quartz, it appears that the conduction mechanism itself is a source of concern $[9,11]$. Assuming the adsorbed layer of water to be continuous it is impossible to conclude whether the observed conductivity is predominantly electronic or ionic. According to eq. (1) with $C=0.05$, we observe that the coverage ratio $R_{\mathrm{c}}$ is (much) smaller than unity for most values of the relative humidity $h$. For example $R_{\mathrm{c}}=0.83,0.17$ and 0.048 when $h=99 \%, 80 \%$ and $50 \%$ respectively. This means that the adsorption occurs on spots with bare regions in between them. Therefore Castagne et al. [9] have described the surface conductivity using the BET theory and the so-called "hopping electron model". They assumed that the surface conductivity is determined by the hopping of electrons between adsorbed spots across bare regions. Starting from these considerations Castagne et al. have derived the following equation for the surface conductivity of $\mathrm{SiO}_{2}$ as a function of the relative humidity:

$\sigma=\sigma_{0} \exp \left[-\alpha\left(\frac{1-h}{C h}\right)^{0.5}\right]$,

where $\sigma_{0}$ is the value of $\sigma$ for $h$ equal to $100 \%$, and $\alpha$ is a constant which depends on the electron mass, the diameter of the adsorbed spots and the energy of electrons on the silicon dioxide surface. The surface conductivity $\sigma$ can also be written as a function of the coverage ratio $R_{\mathrm{c}}$ by using eq. (1):

$\sigma=\sigma_{0} \exp \left[-\alpha\left(\frac{1-R_{\mathrm{c}}}{R_{\mathrm{c}}}\right)^{0.5}\right]$. 
In section 4 we will show that this expression permits us to determine the efficiency of several treatments of the oxide surface in the reduction of the coverage ratio $R_{\mathrm{c}}$. It should be noted that in eq. (3) the contribution of the chemisorbed layer to the surface conductivity has not been taken into account. Due to the observed irreversible behaviour of the chemisorption at room temperature the properties of a chemisorbed surface and thus its conductivity will also be constant. If we wish to account for this we can add a constant term to eq. (3). Experiments, however, have shown that this contribution may be neglected.

\subsection{A description of the oxide-surface potential}

We now consider the surface potential of a silicon dioxide layer contacted on one side with silicon. Assuming the potential of the silicon to be zero and ignoring the thickness of the adsorbed layer, it can be shown that the surface potential $V$ satifies the following partial differential equation:

$$
\frac{\delta V}{\delta t}=\frac{\sigma}{C_{\mathrm{ox}}}\left(\frac{\delta^{2} V}{\delta x^{2}}+\frac{\delta^{2} V}{\delta y^{2}}\right)
$$

with $\sigma$ the surface conductivity of the oxide, $C_{\mathrm{ox}}$ the capacitance per unit area of the oxide, and $x$ and $y$ the directions in which charge motion can take place. The solution of this equation depends on the boundary conditions and initial value of the potential $V$. In the case of a structure which can be considered to be one-dimensional the surface potential can be described by a so-called complementary error function [12]. In all other cases the solution should be approached by means of a numerical procedure.

Considering eq. (4) we observe that for a given potential distribution on the surface, the voltage change per unit of time is linearly dependent on the surface conductivity. Assuming the initial and boundary conditions to remain unchanged this means that, if for a given surface conductivity $\sigma_{i}$ the solution of (4) can be written as $V\left(x, y, t, \sigma_{i}\right)=F(x, y, t)$, for every other value $\sigma_{j}$ the solution satisfies $V\left(x, y, t, \sigma_{j}\right)=F\left(x, y, t^{\prime}\right)$ with $t^{\prime}=t \sigma_{j} / \sigma_{i}$. This indicates that the surface conductivities of different devices can be related to each other by measuring and comparing time intervals.

\section{The experimental method}

In order to measure the surface conductivity by means of surface potential measurements and to qualify the efficiency of different conductivity reduction methods we have applied an open-gate FET structure. This structure is schematically drawn in fig. 2. 
a)

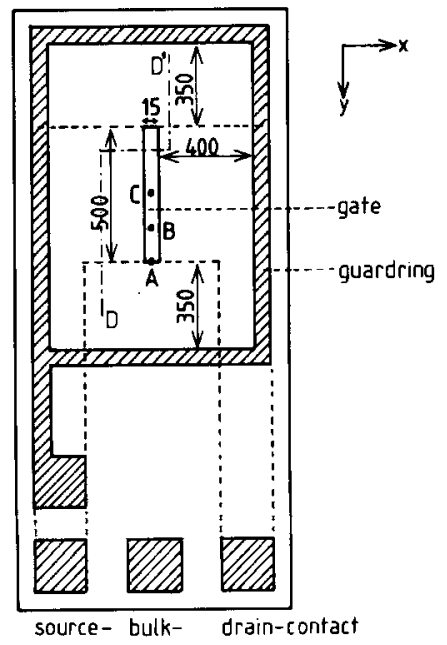

b)

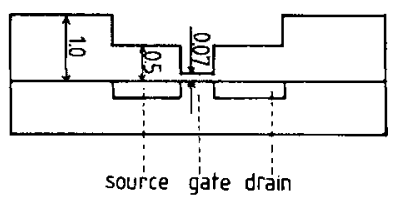

Fig. 2. Schematic drawing of the open-gate FET: (a) top view and (b) cross section. The curve $\mathrm{D}-\mathrm{D}^{\prime}$ in (a) shows where the cross section has been taken. All measures are in $\mu \mathrm{m}$.

The open-gate FET is identical to a MOSFET structure of which the metal gate has been omitted. To define the potential at the edges of the oxide surface a metal guardring is deposited around the gate. Applying a voltage step to this guardring the surface potential inside the ring may change more or less rapidly, depending on the surface conductivity of the oxide-air interface. Surface potential variations inside the gate region cause a change in drain current.

In order to relate drain-current changes to numerical values of the surface conductivity, we have to simulate the potential distribution $V(x, y, t)$ of the oxide-air interface, especially in the gate region, as a function of position $x, y$ and time $t$. Assuming a zero contact potential between guardring and oxide-air interface, the surface potential can be calculated by applying eq. (4) to the structure of fig. 2 . We have resorted to a numerical approach by applying the finite-difference method [13] to eq. (4), yielding a set of finite-difference equations. As can be seen from fig. 2 the oxide thickness is not constant over the whole oxide surface. To account for this effect the finite-difference equation can be modified by varying $C_{\mathrm{ox}}$ appropriately as a function of $x$ and $y$.

Due to the fact that the width $W$ of the channel of the FET is much larger than the length $L$, we assume the surface potential $V$ inside the gate region to be only a function of the $y$ direction, which corresponds to the direction of the width $W$ (see fig. 2). It can be shown that in this case for small values of the 
drain-source voltage $V_{\mathrm{ds}}$ the drain current can be written as:

$I_{\mathrm{d}}=\mu(W / L) C_{\mathrm{ox}}\left[\left(V_{\mathrm{gse}}(t)-V_{\mathrm{t}}\right) V_{\mathrm{ds}}-0.5 V_{\mathrm{ds}}^{2}\right]$,

where $\mu$ is the mobility of the channel charge carriers, $W$ the channel width, $L$ the channel length, $V_{\mathrm{t}}$ the threshold voltage and $V_{\mathrm{gse}}(t)$ the effective gate-source voltage, which can be written as:

$V_{\mathrm{gse}}(t)=W^{-1} \int_{0}^{W} V(y, \bar{t}) \mathrm{d} y$.

Applying these equations to the open-gate FET structure of fig. 2 a simulation program was developed. A detailed description of this program is given by Keskin [14]. Choosing a reasonable value of $10^{-13} \mathrm{~S}$ for the surface conductivity, the surface potential was calculated as a function of time under the following conditions:

$$
\begin{array}{ll}
V \text { (guardring, } t)=1 & \text { for } t>0, \\
V(x, y, 0)=0 & \text { for all } x, y .
\end{array}
$$

The results are given in fig. 3. The curves $\mathrm{A}, \mathrm{B}$ and $\mathrm{C}$ are the surface potential at three places $\mathrm{A}, \mathrm{B}$ and $\mathrm{C}$ over the gate region of the FET, as indicated in fig. 2. Curve $\mathrm{D}$ is the numerically approached effective gate-source potential. Considering eq. (5) we note that the drain current is a linear function of the effective gate-source potential, as drawn in fig. 3, curve D.

Using the open-gate FET structure the drain current can be measured as a function of time by means of the experimental set-up shown in fig. 4. All experiments described in this chapter have been performed at room temperature and $100 \%$ relative humidity, which was achieved by placing the open-gate FET structure in a closed box, partly filled with water. Under these conditions

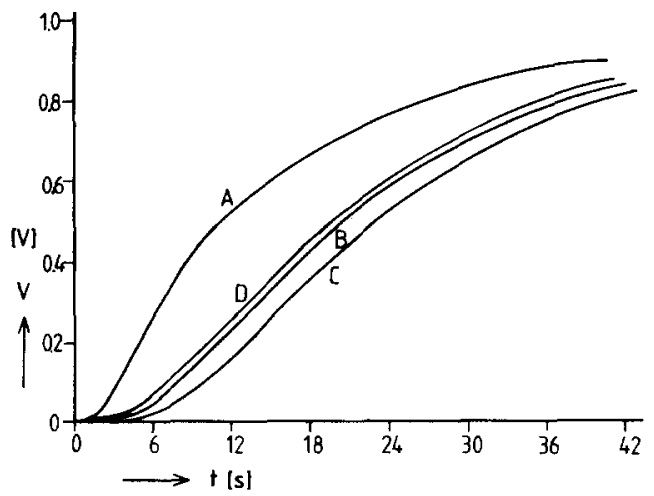

Fig. 3. Simulated potential at three places A, B and C on the oxide surface, as indicated in fig. 2, after applying a voltage step to the guardring, with $\sigma=10^{-13} \mathrm{~S}$. Curve $\mathrm{D}$ is the calculated effective gate-source voltage. 


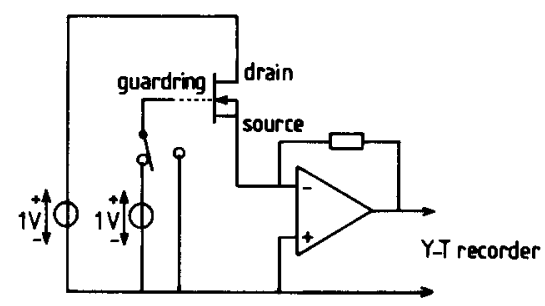

Fig. 4. Experimental set-up for measuring drain-current changes after applying a voltage step to the guardring.

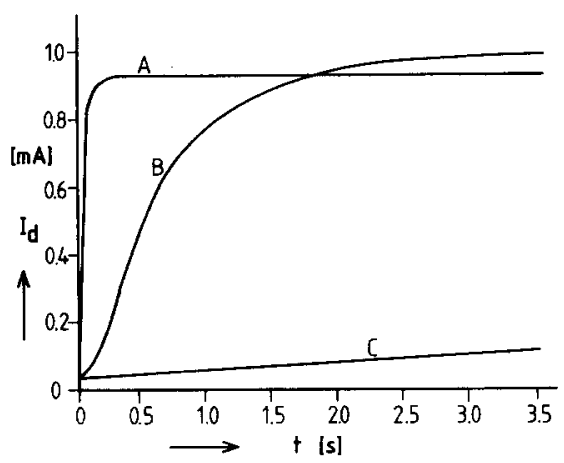

Fig. 5. Typical measured drain current as a function of time after applying a voltage step to the guardring of the test-structure, treated by several means: (A) untreated, (B) deionized water and (C) HMDS.

experiments can be completed within a reasonable time. We assume that with lower values of the relative humidity, the results will not differ except for the time scale.

The typical behaviour of the measured drain current as a function of time after applying a voltage step, for untreated, not cleaned devices is shown in fig. 5 (curve A). Using the simulation program we calculated the behaviour of the drain current as a function of time for different values of the surface conductivity. Fitting these results with the experimental curve A of fig. 5 we found that the approximated surface conductivity is $10^{-10} \mathrm{~S}$.

\section{Experimental results}

Considering the theoretical description of section 2, several methods can be suggested to lower the surface conductivity. The value of $\sigma_{0}$ may depend on the presence of contaminating ions. Furthermore, according to eq. (3), we expect an exponential dependence of the surface conductivity on the coverage 
ratio $R_{\mathrm{c}}$ of the surface. The coverage ratio of an untreated oxide surface in an ambient with $100 \%$ relative humidity is equal to unity, according to eq. (1). It can be expected that a chemical surface modification of the oxide surface reduces the adsorption of water vapour and thus the coverage ratio for a given value of the relative humidity. In that case eq. (1) will not be valid. However, eq. (3) can still be used to calculate the value of the coverage ratio after treatment. Two methods seem now to be usable for reducing the surface conductivity:

(1) Cleaning of the surface

(2) Chemical surface modification.

\subsection{Cleaning of the surface}

The cleaning of an oxide surface by rinsing with an appropriate solution, as for instance mentioned by Shockley et al. [6], is a simple method. They suggested that treatment with solvents having a low dipole moment and a low dissociation constant would lower the surface conductivity. We have found that even a treatment of the surface with deionized water, by which the density of contaminants like $\mathrm{Na}^{+}$and $\mathrm{K}^{+}$can be lowered, may result in a lower value of the surface conductivity. The measured drain current of a cleaned device as a function of time, after applying a voltage step to the guardring, is given in fig. 5, curve B. The approximated surface conductivity appears to be about $10^{-12} \mathrm{~S}$. This value corresponds rather well with the value of about $10^{-13} \mathrm{~S}$ at 96\% relative humidity, reported by Awakuni and Calderwood [8]. Comparing the value of the surface conductivity of our devices before and after treatment we observe a reduction with a factor 100 . We have also tried to reduce the surface conductivity of these cleaned devices by rinsing with for instance toluene and hexane. In that case however we did not observe a further reduction of the surface conductivity.

\subsection{Chemical surface modification}

Considering the adsorption mechanisms for water vapour on silicon dioxide, we expected that a reduction of the number of silanol groups on the surface by means of an appropriate chemical modification would result in a reduced surface conductivity.

Yanazawa et al. have shown that several agents, such as hexamethyldisilazane (HMDS) and trimethylchlorosilane (TMS), convert the surface from hydrophilic into hydrophobic [15]. It is also known that the pH-sensitivity of a liquid-oxide interface depends on the number of silanol groups on the oxide surface [16]. Van den Berg et al. have recently investigated in which way the $\mathrm{pH}$-sensitivity of the ion sensitive field-effect transistor (ISFET) can be controlled by chemical modification of the $\mathrm{SiO}_{2}$ surface [17], reducing the 
density of silanol groups per unit are. They have used silanizing agents and found that they reduce the $\mathrm{pH}$-sensitivity of the $\mathrm{SiO}_{2}$ surface considerably.

Regarding these results we also expect a reduction of the surface conductivity by treatment of the surface with silanizing agents. The following approximate reduction factors have been obtained by means of a liquid phase reaction at $75^{\circ} \mathrm{C}$ :

$\begin{array}{llr}\text { hexamethyldisilazane } & \text { (HMDS) } & 1000, \\ \text { dichlorodimethylsilane } & \text { (DCDMS) } & 10000, \\ \text { trimethoxyvinylsilane } & \text { (TMVS) } & 100, \\ \text { triethoxymethylsilane } & \text { (TEMS) } & 40 .\end{array}$

In this case we have compared the surface conductivity at $100 \%$ relative humidity of devices which have been rinsed with deionized water, before and after treatment with a silanizing agent. We observe that especially DCDMS and HMDS seem to be very promising.

The drain current for a HMDS-treated device as a function of time, after applying a voltage step to the guardring is shown in fig. 5, curve C. The approximated value of the surface conductivity of this device is about $10^{-15} \mathrm{~S}$.

With eq. (3) we can now estimate the relative number of surface silanol groups which have been passivated. In the case of an untreated device the coverage ratio is equal to unity at $100 \%$ relative humidity according to eq. (1). For a treated device it will be smaller than unity. Considering the experimental results, presented by Castagne et al. [9], the value of $\alpha$ can be calculated to be 1.63 for $C=0.05$. Assuming $\sigma_{0}$ and $\alpha$ to be unchanged by the surface modification and using the measured reduction factors for HMDS and DCDMS, the coverage ratio can now be calculated. We obtain $R_{\mathrm{c}}=0.05$ and 0.03 for a HMDS- and DCDMS-treated surface respectively. This means that $95 \%$ and $97 \%$ of the original silanol groups are passivated. Van den Berg et al. [17] have calculated the $\mathrm{pH}$-sensitivity of silicon dioxide as a function of the number of silanol groups at its surface. They have also measured the $\mathrm{pH}$-sensitivity of HMDS-treated $\mathrm{SiO}_{2}$. Comparing their experimental and theoretical results we conclude that between $90 \%$ and $99 \%$ of the original silanol groups have been passivated by the chemical treatment. This corresponds very well with our results.

In the case of HMDS the $\mathrm{Si}-\mathrm{OH}$ group is replaced by an $\mathrm{Si}-\mathrm{O}-\mathrm{Si}-\left(\mathrm{CH}_{3}\right)_{3}$ group, and in the case of DCDMS an $(\mathrm{Si}-\mathrm{O})_{2}-\mathrm{Si}-\left(\mathrm{CH}_{3}\right)_{2}$ group is formed. It is suggested that due to so-called steric hindrance of these rather large molecules it would be impossible to replace all $\mathrm{Si}-\mathrm{OH}$ groups. Due to the fact however that not every $\mathrm{Si}$ atom at the oxide surface forms an $\mathrm{Si}-\mathrm{OH}$ group [16], we do not expect that steric hindrance severly limits the efficiency of this method, but maybe it declares the $95 \%$ and $97 \%$ efficiency of the HMDS and DCDMS treatment respectively. 


\section{Conclusions}

Using the BET-adsorption theory and the so-called hopping electron theory presented by Castagne et al. [9] we found a relationship between the surface conductivity, relative humidity and coverage ratio of a surface with water.

Applying the theoretical description, presented in section 2, we developed a computer program by which the drain current of the open-gate FET can be simulated. Fitting theoretical results and experimental curves the surface conductivity of silicon dioxide can be determined. In this way we found that the surface conductivity of devices, not cleaned with deionized water, is about $10^{-10} \mathrm{~S}$ in air with a relative humidity of $100 \%$.

The approximated value of the surface conductivity of cleaned devices appeared to be about $10^{-12} \mathrm{~S}$, which is in rather good agreement with the value of $10^{-13} \mathrm{~S}$ measured by Awakuni and Calderwood in an ambient with $96 \%$ relative humidity [8]. Comparing the value of the surface conductivity before and after cleaning of the surface we observed a reduction by a factor 100. The surface conductivity of HMDS-treated devices appeared to be about $10^{-15} \mathrm{~S}$, which is 1000 times smaller as compared to untreated devices.

Assuming that a reduction of the surface conductivity due to a chemical modification of the surface can be regarded as a reduction of the number of silanol groups, the theory was used to quantify the effect of different chemical treatments. The HMDS treatment for instance yielded a reduction of $95 \%$ of the original silanol groups. This result appeared to correspond very well with that described by van den Berg et al. [17].

Considering the experimental results we conclude that the chemical modification by means of silanizing agents seems to be a promising tool for the reduction of the surface conductivity of silicon dioxide. Most probably the effect of this method can even be improved by optimizing the processing parameters such as the temperature at which the chemical reaction is performed.

\section{Acknowledgements}

The authors wish to thank Professor O.W. Memelink, Dr. H. Wallinga and Ir. A. van den Berg for their helpful suggestions and stimulating discussions.

\section{References}

[1] J.A. Voorthuyzen and P. Bergveld, IEEE Trans. Electron Devices ED-32 (1985) 1185.

[2] H.C. Natanson, W.E. Newell, R.A. Wickstrom and J.R. Davis, IEEE Trans. Electron Devices ED-14 (1967) 117.

[3] M.N. Horenstein, IEEE Trans. Electron Devices ED-32 (1985) 716. 
[4] M. Esashi, H. Komatsu and T. Matsuo, Sensors Actuators 4 (1983) 544.

[5] A. Yoshikawa and T. Suzuki, J. Acoust. Soc. Am. 64 (1978) 725.

[6] W. Shockley, W.W. Hooper, H.J. Queisser and W. Schroen, Surface Sci. 2 (1964) 277.

[7] K. Haufe and S.R. Morrison, Adsorption (De Gruyter, Berlin, 1974) p. 13.

[8] Y. Awakuni and J.H. Calderwood, J. Phys. D (Appl. Phys.) 5 (1972) 1038.

[9] R. Castagne, P. Hesto and A. Vapaille, Thin Solid Films 17 (1973) 253.

[10] G.J. Young, J. Colloid Sci. 13 (1958) 67.

[11] A.D. Martin and K.J. McLean, J. Appl. Phys. 48 (1977) 2950.

[12] E.S. Schlegel, G.L. Schnable, R.F. Schwarz and J.P. Spratt, IEEE Trans. Electron Devices ED-15 (1968) 973.

[13] G.D. Smith, Numerical Solutions of Partial Differential Equations (Oxford Univ. Press, London, 1971) p. 41.

[14] K. Keskin, Report No. 357, Bio-Information Group, Twente University of Technology, The Netherlands, 1986.

[15] H. Yanazawa, H. Utsigi, N. Hashimoto and M. Ashikawa, Proc. 6th Intern. Vacuum Congr. 1974, Japan. J. Appl. Phys. Suppl. 2 (1974) 753.

[16] L. Bousse, N.F. de Rooij and P. Bergveld, IEEE Trans. Electron Devices ED-30 (1983) 1263.

[17] A. van den Berg, P. Bergveld, D.N. Reinhoudt and E.R. Sudholter, Sensors Actuators 8 (1985) 129. 